University of Wollongong

Research Online

Faculty of Engineering and Information

Faculty of Engineering and Information

Sciences - Papers: Part A

Sciences

$1-1-2013$

\title{
Degradation of azo dye acid orange 7 in a membrane bioreactor by pellets and attached growth of Coriolus versicolour
}

\author{
Faisal I. Hai \\ University of Wollongong, faisal@uow.edu.au \\ Kazuo Yamamoto \\ University Of Tokyo \\ Fumiyuki Nakajima \\ University Of Tokyo \\ Kensuke Fukushi \\ University Of Tokyo \\ Long D. Nghiem \\ University of Wollongong, longn@uow.edu.au
}

See next page for additional authors

Follow this and additional works at: https://ro.uow.edu.au/eispapers

Part of the Engineering Commons, and the Science and Technology Studies Commons

Research Online is the open access institutional repository for the University of Wollongong. For further information contact the UOW Library: research-pubs@uow.edu.au 


\title{
Degradation of azo dye acid orange 7 in a membrane bioreactor by pellets and attached growth of Coriolus versicolour
}

\begin{abstract}
The aim of this study was to systematically compare the degradation of azo dye acid orange 7 by spongy pellets and attached biofilm of Coriolus versicolour (NBRC 9791) in a membrane bioreactor (MBR) under non-sterile conditions. Mild stirring $(35 \mathrm{rpm})$ resulted in spherical $(\varphi=0.5 \mathrm{~cm})$, spongy pellets and concomitantly triggered high enzymatic activity of the fungus, allowing for excellent decolouration ( $>99 \%)$ of a synthetic wastewater containing the dye. However, bacterial contamination eventually damaged the fungus pellets, leading to decreased decolouration efficiency. Promotion of attached growth on a plastic support along with formation of spherical spongy pellets allowed maintenance of high enzymatic activity and decolouration/degradation for an extended period. Hydraulic retention time (HRT) could influence the level of enzymatic activity and decolouration; however, even at the shortest HRT (1 day) examined, the MBR could accomplish $>95 \%$ decolouration. (C) 2013 Elsevier Ltd.
\end{abstract}

\section{Keywords}

pellets, attached, growth, coriolus, degradation, versicolour, azo, dye, acid, orange, 7, membrane, bioreactor

Disciplines

Engineering | Science and Technology Studies

\section{Publication Details}

Hai, F. I., Yamamoto, K., Nakajima, F., Fukushi, K., Nghiem, L. D., Price, W. E. \& Jin, B. (2013). Degradation of azo dye acid orange 7 in a membrane bioreactor by pellets and attached growth of Coriolus versicolour. Bioresource Technology, 141 29-34.

\section{Authors}

Faisal I. Hai, Kazuo Yamamoto, Fumiyuki Nakajima, Kensuke Fukushi, Long D. Nghiem, William E. Price, and Bo Jin 


\title{
Degradation of azo dye acid orange 7 in a membrane bioreactor by pellets and attached growth of Coriolus versicolour
}

\author{
Faisal I. Hai ${ }^{\text {a,b,*, Kazuo Yamamoto }}{ }^{\text {b }}$, Fumiyuki Nakajima ${ }^{b}$, Kensuke Fukushi ${ }^{\text {c }}$, Long D. \\ Nghiem ${ }^{\text {a }}$, William E. Price ${ }^{\mathrm{d}}$, and Bo Jin ${ }^{\mathrm{e}}$
}

${ }^{a}$ Strategic Water Infrastructure Laboratory, School of Civil, Mining and Environmental Engineering, University of Wollongong, NSW 2500, Australia.

${ }^{\mathrm{b}}$ Environmental Science Center, The University of Tokyo, Tokyo 113-0033, Japan.

${ }^{\mathrm{c}}$ Integrated Research System for Sustainability Science, The University of Tokyo, Tokyo 113-8654, Japan.

${ }^{\mathrm{d}}$ Strategic Water Infrastructure Laboratory, School of Chemistry, University of Wollongong, NSW 2500, Australia.

${ }^{\mathrm{e}}$ School of Chemical Engineering, The University of Adelaide, Adelaide, SA 5005, Australia.

* Corresponding author

Faisal I. Hai, Strategic Water Infrastructure Laboratory, School of Civil, Mining and Environmental Engineering, University of Wollongong, NSW 2500, Australia (E-mail: faisal@,uow.edu.au, Phone : +612-4221-3054)

Faisal I. Hai, Kazuo Yamamoto, Fumiyuki Nakajima, Kensuke Fukushi, Long D. Nghiem, William E. Price, Bo Jin. Degradation of azo dye acid orange 7 in a membrane bioreactor by pellets and attached growth of Coriolus versicolour. Bioresource Technology, Volume 141, August 2013, Pages 29-34 


\begin{abstract}
The aim of this study was to systematically compare the degradation of azo dye acid orange 7 by spongy pellets and attached biofilm of Coriolus versicolour (NBRC 9791) in a membrane bioreactor (MBR) under non-sterile conditions. Mild stirring $(35 \mathrm{rpm})$ resulted in spherical $(\varphi=0.5 \mathrm{~cm})$, spongy pellets and concomitantly triggered high enzymatic activity of the fungus, allowing for excellent decolouration (>99\%) of a synthetic wastewater containing the dye. However, bacterial contamination eventually damaged the fungus pellets, leading to decreased decolouration efficiency. Promotion of attached growth on a plastic support along with formation of spherical spongy pellets allowed maintenance of high enzymatic activity and decolouration for an extended period. Hydraulic retention time (HRT) could influence the level of enzymatic activity and decolouration; however, even at the shortest HRT (1 d) examined, the MBR could accomplish $>95 \%$ decolouration.
\end{abstract}

Keywords: azo dye; decolouration; morphology; membrane bioreactor (MBR); non-sterile; white-rot fungi.

Faisal I. Hai, Kazuo Yamamoto, Fumiyuki Nakajima, Kensuke Fukushi, Long D. Nghiem, William E. Price, Bo Jin. Degradation of azo dye acid orange 7 in a membrane bioreactor by pellets and attached growth of Coriolus versicolour. Bioresource Technology, Volume 141, August 2013, Pages 29-34 


\section{Introduction}

Dye effluent contains chemicals, including dye itself, that are toxic to various microbiological and fish species, and can also cause human health disorders and severe damage to important human organs. Synthetic dyes with complex structures deriving from the use of different chromophoric groups can be extremely recalcitrant (Hai et al., 2007). Unlike the bacteria in conventional wastewater treatment systems, aerobic white-rot fungi (WRF) can degrade wide varieties of resistant compounds including textile dyes by non-specific extracellular enzymes (Wesenberg et al., 2003). However, contrary to the performance shown in aseptic batch tests, the application of WRF in continuous bioreactors for dye wastewater treatment has been so far impeded by problems such as excessive growth of fungi causing reactor-clogging (Zhang et al., 1999), bacterial contamination inhibiting fungal decolouration (Hai et al., 2009; Libra et al., 2003), and loss of the extracellular enzymes and mediators essential for dye degradation with treated effluent (Hai et al., 2012). Of a major concern is the inhibition of enzymatic activity and decolouration performance due to bacterial contamination. WRF grows slowly compared with bacteria. Once bacteria invade the system, bacteria can compete with WRF for substrate and can thus inhibit fungal growth, rendering the WRF system eventually ineffective for dye decolouration and degradation (Hai et al., 2009; Libra et al., 2003).

The approaches that have been used to date to improve fungal decolouration under non-sterile environment include use of low $\mathrm{pH}$ and nitrogen-limited medium, omission of certain trace elements (e.g., iron) from the culture medium, periodic addition of fresh biomass, de-coupling of growth (sterile condition) and decolourization (non-sterile condition) stages, and use of bactericide (e.g. ozone) to control the bacterial contamination(Blanquez et al., 2008; Gao et al., 2006; Libra et al., 2003; Zhou \& Wen, 2009). None of these approaches on their own has been proven to be a long-term solution to the problem of bacterial contamination, and further research is deemed imperative.

Faisal I. Hai, Kazuo Yamamoto, Fumiyuki Nakajima, Kensuke Fukushi, Long D. Nghiem, William E. Price, Bo Jin. Degradation of azo dye acid orange 7 in a membrane bioreactor by pellets and attached growth of Coriolus versicolour. Bioresource Technology, Volume 141, August 2013, Pages 29-34 
Different morphological forms of fungi can show different characteristics such as specific growth rate and enzyme secretion: one form may include several physiological states of fungi with the production of specific metabolites, which may not occur in another form. The effects of fungal morphology on fine chemical synthesis by pure fungal cultures has been well documented (Papagianni, 2004); however, very few articles have specifically considered the effects of morphology on the degree of removal of recalcitrant pollutants such as dye. Furthermore, studies available to date have focused mostly on a single type of morphologies (e.g., dispersed filaments, spherical pellets and attached biofilm) under aseptic conditions. For example, Bermek et al. (2004) reported that growth in dispersed filaments can increase the viscosity of the medium (low oxygen levels), limiting the mass transfer, causing a lower ligninolytic enzyme production. Borras et al. (2008) demonstrated high degree of dye decolourization using the ligninolytic fungus Trametes versicolor in pellet form, however, the performance was not compared with other morphological forms. Notably, compared with the morphologies encountered in suspended cultures (e.g., dispersed filaments and spherical pellets), immobilized cultures (entrapment into some matrix or attached growth onto some supports) tend to show a higher level of enzymatic activity and more resilience to environmental perturbations such as shear damage and $\mathrm{pH} /$ toxic shock (Rodriguez Couto, 2009). For example, Zhang et al. (1999) observed better decolouration of an azo dye by alginate-immobilised fungus than dispersed filaments in different reactor configurations; however, direct comparison with pellet morphology was not performed in their study.

A range of techniques for pellet formation (Erdal \& Taskin, 2006; Yang et al., 2008; Žmak et al., 2006) and carrier materials for fungal immobilization (Rodriguez Couto, 2009) have been explored with encouraging dye removal performance; however, an important area of research requiring greater focus is the long term operation of bioreactors with pellets or attached growth fungi under non-sterile conditions. A comprehensive literature review could identify only a few studies where the removal performance of bioreactors containing pellets or attached growth fungi under non-sterile conditions has been investigated (Leidig et al., 1999; Nilsson et al., 2006; Tang et al., 2011). A further notable omission in the literature is a systematic comparison between different morphological forms under non-sterile conditions and exploration of their combinations Faisal I. Hai, Kazuo Yamamoto, Fumiyuki Nakajima, Kensuke Fukushi, Long D. Nghiem, William E. Price, Bo Jin. Degradation of azo dye acid orange 7 in a membrane bioreactor by pellets and attached growth of Coriolus versicolour. Bioresource Technology, Volume 141, August 2013, Pages 29-34 
(e.g., pellets and attached growth) to effectively prevent bacterial inhibition of dye decolouration/degradation.

The advantages of membrane bioreactors (MBR) such as maintenance of high WRF concentration and prevention of enzyme washout have been demonstrated in our previous studies (Hai et al., 2006; Hai et al., 2012). In line with the research gaps delineated above, the aim of the current study was to systematically compare the enzymatic activity and dye degradation performance of two types of morphology of Coriolus versicolour (NBRC 9791), namely, spongy pellets and attached biofilm, in MBR under non-sterile conditions and propose strategies to achieve stable dye removal. Performance in sterile batch tests was assessed to form a basis of their performance comparison during non-sterile operation.

\section{Materials and methods}

\subsection{White-rot fungus, dye and synthetic wastewater}

The white-rot fungus Coriolus versicolour, NBRC 9791 obtained from the NITE Biological Resource Centre (NBRC), Japan was used for this study. Decolouration/degradation of acid orange 7 (Sigma-Aldrich Co., USA), which is a low molecular weight (350 g/mol), monoazo dye, was monitored. This is a water soluble dye and produces orange colour (peak absorbance at $481 \mathrm{~nm})$. A synthetic wastewater was prepared by adding dye $(100 \mathrm{mg} / \mathrm{L})$ and $\operatorname{starch}(2 \mathrm{~g} / \mathrm{L})-$ two common components in real textile wastewater - and other nutrients into Milli-Q water. The other components of the synthetic wastewater were as follows: $0.1 \mathrm{~g} / \mathrm{L}$ urea, $2 \mathrm{~g} / \mathrm{L} \mathrm{KH}_{2} \mathrm{PO}_{4}$, $0.099 \mathrm{~g} / \mathrm{L} \mathrm{CaCl}_{2}, 1.025 \mathrm{~g} / \mathrm{L} \mathrm{MgSO}_{4} \cdot 7 \mathrm{H}_{2} \mathrm{O}, 0.001 \mathrm{~g} / \mathrm{L}$ thiamine and $1 \mathrm{~mL} / \mathrm{L}$ trace elements. Stock trace elements solution was prepared by dissolving $0.125 \mathrm{~g} \mathrm{CuSO}_{4} \cdot 5 \mathrm{H}_{2} \mathrm{O}, 0.05 \mathrm{~g} \mathrm{H}_{2} \mathrm{MoO}_{4}, 0.061$ g $\mathrm{MnSO}_{4} \cdot 5 \mathrm{H}_{2} \mathrm{O}, 0.043 \mathrm{~g} \mathrm{ZnSO}_{4} \cdot 7 \mathrm{H}_{2} \mathrm{O}, 0.082 \mathrm{~g} \mathrm{Fe}_{2}\left(\mathrm{SO}_{4}\right)_{3} \cdot 14 \mathrm{H}_{2} \mathrm{O}$ in $1 \mathrm{~L}$ of Milli-Q water. With this composition, the final $\mathrm{pH}$ of the solution was 4.5. For batch tests described in Section 2.2, the above-mentioned same medium (without dye) was used as the culture medium.

\subsection{Batch test protocol}

Faisal I. Hai, Kazuo Yamamoto, Fumiyuki Nakajima, Kensuke Fukushi, Long D. Nghiem, William E. Price, Bo Jin. Degradation of azo dye acid orange 7 in a membrane bioreactor by pellets and attached growth of Coriolus versicolour. Bioresource Technology, Volume 141, August 2013, Pages 29-34 
Before assessing the enzymatic activity and decolouration performance of the fungus in suspended (pellet) and attached form during non-sterile operation of the reactors, the enzymatic activity of C. versicolour (NBRC 9791) in case of suspended and attached growth was assessed in sterile batch tests. Two forms of suspended biomass, namely, dispersed filaments and spherical spongy pellets, were assessed. On the other hand, attached growth was established on 1 $\mathrm{cm}$ pieces of flexible plastic tubing (Masteflex Tygon Lab Tubing) as carriers. To facilitate interconnected attached growth over the surface and within the tube-lumen, the surface of the tubing pieces were roughened using a rasp file, and a $0.25 \mathrm{~cm}$ hole was punched at the centre of each piece.

The inoculant for the batch tests was prepared by disintegrating freshly grown and enzyme secreting large mycelial clumps of the fungus into dispersed filaments by sonication (Branson sonifier 450, USA) for 5 minutes under sterile conditions (Hai et al., 2009). Development of different fungal morphologies depended on the intensity of agitation (shear rate) during incubation. For the suspended cultures, the biomass resulting from the above step $(0.015 \mathrm{~g}$ dry weight) was incubated aseptically at the optimum growth temperature of $28{ }^{\circ} \mathrm{C}$ for two weeks in $500 \mathrm{~mL}$ (spongy pellets) and $1000 \mathrm{~mL}$ (dispersed filaments) flasks, each containing $100 \mathrm{~mL}$ culture medium, on a reciprocal shaker (BR-300LF, Taitec bio-shaker, Japan) at 120 cycle/min. For the attached growth, the same amount of biomass as above was incubated in $500 \mathrm{~mL}$ flasks containing $100 \mathrm{~mL}$ medium and 10 tubing pieces as the carriers at a shaking speed of 70 cycle/min.

\subsection{Reactor set-up and operation protocol}

As noted earlier, the advantages of MBR such as maintenance of high WRF concentration and prevention of enzyme washout have been demonstrated in our previous studies (Hai et al., 2006; Hai et al., 2012; Hai et al., 2009). Therefore, also in this study MBR was chosen as the reactor system. Because the presence of a membrane module within the reactor can potentially lead to formation of attached growth on the module (Gao et al., 2009; Hai et al., 2006), in order to allow observation of the performance of the spongy pellets only, no membrane was used in the first reactor (Section 2.3.1). Both reactors were initially operated in sequencing batch mode to Faisal I. Hai, Kazuo Yamamoto, Fumiyuki Nakajima, Kensuke Fukushi, Long D. Nghiem, William E. Price, Bo Jin. Degradation of azo dye acid orange 7 in a membrane bioreactor by pellets and attached growth of Coriolus versicolour. Bioresource Technology, Volume 141, August 2013, Pages 29-34 
facilitate the formation of spongy pellets as per previous reports (Liu \& Tay, 2004; Papagianni, 2004).

\subsubsection{Reactor with fungal pellet}

A cylindrical glass container $(15 \mathrm{~cm}$ diameter $\times 22 \mathrm{~cm}$ height $)$ containing the synthetic wastewater $(1.5 \mathrm{~L})$ was inoculated with $0.5 \mathrm{~g}$ (dry weight) freshly grown, elliptical mycelial clumps (approximately $2 \times 4 \mathrm{~cm}$ ). The medium was then subject to mild stirring (35 rpm) on a jar-tester (Sugiyama Gen Co. Ltd., Japan) equipped with a $6 \mathrm{~cm}$ flat plastic pedal. Continuous operation in sequencing batch mode under different hydraulic retention time (HRT), as detailed later, was started one week after the inoculation; by then the initial large clumps had been disintegrated and $0.5 \mathrm{~cm}$ spherical spongy pellets were obtained. The reactor medium at this point exhibited an enzymatic activity of approximately $40 \mu \mathrm{M} / \mathrm{min}$ (measured in reference to 2,6-dimethoxy phenol, see Section 2.4).

During the 'draw' period ( $5 \mathrm{~min}$ ), the stirrer was turned off, the mixed liquor was allowed to settle and the treated supernatant was decanted off. During the following 'fill' period (1 min), new medium was poured into the reactor manually. However, to prevent loss of biomass that escaped with supernatant, the withdrawn treated medium was filtered ( $1 \mu \mathrm{m}$ glass fiber filter, Advantec) and retained biomass was returned to the reactor. The synthetic wastewater was autoclaved only at initial inoculation. The reactor was operated under an HRT of 1-3 d during the first four weeks. Because severe bacterial contamination occurred on the fourth week, to avoid reduction in colour removal, the reactor was operated at an HRT of $4.5 \mathrm{~d}$ (withdrawal/addition of $67 \%$ of the reactor medium every third day) for the rest of the period (until Day 90).

\subsubsection{Membrane bioreactor with suspended (pellet) and attached growth}

Similar to the reactor with fungal pellets, a cylindrical glass container $(15 \mathrm{~cm}$ diameter x $22 \mathrm{~cm}$ height) was used for the MBR. Compared with the first reactor, two modifications were carried out in this reactor to facilitate attached growth: i) two plastic tubes were inserted up to the bottom of the reactor and wastewater was introduced through them (these tubes were the same type as used in the batch test, and were modified in the same way, i.e., roughened surface and a Faisal I. Hai, Kazuo Yamamoto, Fumiyuki Nakajima, Kensuke Fukushi, Long D. Nghiem, William E. Price, Bo Jin. Degradation of azo dye acid orange 7 in a membrane bioreactor by pellets and attached growth of Coriolus versicolour. Bioresource Technology, Volume 141, August 2013, Pages 29-34 
$0.25 \mathrm{~cm}$ punch every $\mathrm{cm})$; ii) a small (fibre length $5.5 \mathrm{~cm})$, microfiltration $(0.4 \mu \mathrm{m})$ polyethylene (hydrophilized) hollow fibre membrane module (Mitsubishi Rayon, Japan) was inserted in the reactor. The inoculation methods were the same as in the first reactor. Following the operation in sequencing batch mode $(\mathrm{HRT}=3-4.5 \mathrm{~d})$, the MBR was operated in continuous flow mode $($ HRT $=4.5-1 \mathrm{~d})$ for the initial 40 days. The membrane module, which had a total surface area of $0.256 \mathrm{~m}^{2}$, was operated at a flow rate of $5.21 \mathrm{~mL} / \mathrm{min}$ with a $6 \mathrm{~min}$ on and $24 \mathrm{~min}$ off cycle, thereby imposing a small average flux and instantaneous flux of 0.0059 and $0.0292 \mathrm{~m} / \mathrm{d}$, respectively. With such a small operating flux, the transmembrane pressure remained stable throughout the observation period without any cleaning, thus allowing the focus of the study to remain on fungal dye removal performance.

\subsection{Analytical methods}

TOC was measured with a total organic carbon analyser (TOC-V, Shimadzu, Japan). Colour measurements of samples at the peak wavelength of the dye $(481 \mathrm{~nm})$ were carried out using a spectrophotometer (U-2010, Hitachi, Japan). In addition to colour measurements, a HPLC

system (HP Agilnet 1100, USA), equipped with a Spherisorb ODS2 column (200 x 4.6-mm, 5 $\mu \mathrm{m}$ pore size) and a diode array detector was used to monitor dye degradation. The column temperature was $35^{\circ} \mathrm{C}$, the sample injection volume was $100 \mu \mathrm{L}$, and the peaks were monitored at 481 and $254 \mathrm{~nm}$. Each sample was analyzed for a period of 15 minutes, and acetonitrile was used as the eluent.

Under the conditions tested, the collected fungus strain exhibited extracellular secretion of the enzyme laccase. Laccase activity was measured by monitoring the change in absorbance at 468 $\mathrm{nm}$ due to the oxidation of 2,6-dimethoxy phenol at room temperature over 2 min using a spectrophotometer. Laccase activity was calculated from the molar extinction coefficient $\varepsilon=$ 49.6/mM/cm and expressed in $\mu \mathrm{M}$ substrate $/ \mathrm{min}$.

\section{Results and discussion}

\subsection{Enzymatic activity of suspended and attached growth biomass}

Faisal I. Hai, Kazuo Yamamoto, Fumiyuki Nakajima, Kensuke Fukushi, Long D. Nghiem, William E. Price, Bo Jin. Degradation of azo dye acid orange 7 in a membrane bioreactor by pellets and attached growth of Coriolus versicolour. Bioresource Technology, Volume 141, August 2013, Pages 29-34 
In this study, during sterile batch tests, the attached growth biomass of $C$. versicolour demonstrated relatively better and more stable enzymatic activity. In case of the suspended biomass, the spherical spongy pellets outperformed the dispersed filaments. The enzymatic activity in the test solution after $10 \mathrm{~d}$ in case of the attached growth biomass, spongy pellets and dispersed filaments was approximately 25, 15 and $10 \mu \mathrm{M} / \mathrm{min}$, respectively (measured in reference to 2,6-dimethoxy phenol). Our results are in good agreement with the available reports. Pellet formation compared with growth in dispersed filaments, has been reported to be more suitable for high enzymatic activity (Bermek et al., 2004; Jimenez-Tobon et al., 1997). This has been explained by hindered mass transfer due to the increased viscosity in the culture medium (low oxygen levels) in presence of a significant amount of free mycelia filaments. On the other hand, several studies have confirmed higher enzymatic activity and/or decolouration performance of immobilised/attached growth biomass compared with free mycelia (Rodriguez Couto, 2009). The batch test data validated the choice of spherical pellets and attached growth biomass for further testing and formed a basis of their performance-comparison during nonsterile operation of the fungal reactors.

\subsection{Performance of the fungal reactor with suspended spongy pellets}

Upon establishment of the spherical spongy pellets of approximately $0.5 \mathrm{~cm}$ diameter, the reactor was operated under different HRT in a sequencing batch mode. The pellets were easily settleable, demonstrated high enzymatic activity in the reactor and achieved excellent decolouration (Figure 1). Despite the fact that no specific measures to prevent bacterial contamination such as sterilisation of the wastewater, were adopted, no significant bacterial contamination was noticed for approximately four weeks. This compares favourably with previous reports where immediate contamination and disappearance of enzymatic activity was noticed in reactors with dispersed filaments (Hai et al., 2009). During this period, a clear instant influence of HRT on the level of laccase activity in the reactor was observed; however, the reactor accomplished excellent decolouration even under the shortest HRT explored (1 day) (Figure 1). Disappearance and significant reduction of the peaks associated with the dye in visible $(481 \mathrm{~nm})$ and UV $(254 \mathrm{~nm})$

Faisal I. Hai, Kazuo Yamamoto, Fumiyuki Nakajima, Kensuke Fukushi, Long D. Nghiem, William E. Price, Bo Jin. Degradation of azo dye acid orange 7 in a membrane bioreactor by pellets and attached growth of Coriolus versicolour. Bioresource Technology, Volume 141, August 2013, Pages 29-34 
wavelengths, respectively in HPLC-DAD analysis confirmed significant degradation of the aromatic structure along with the colour producing chromophoric group of the dye .

\section{Figure 1}

In the course of operation under non-sterile environment, however, severe bacterial contamination could be observed from day 26 (Figure 1). Significantly reduced decolouration performance and highly turbid appearance of the reactor medium provided preliminary indication of the bacterial contamination, which was verified by microscopic observation (not shown). Of special interest was the subsequent gradual revival of the decolouration performance and lessening of bacterial contamination (Figure 1, beyond Day 45). Although fungal laccase has been comprehensively shown to be involved in the degradation of resistant compounds, it may be involved in a wider range of other physiological functions and may be triggered by different kinds of stresses (Baldrian, 2004). Perhaps WRF use extracellular enzymes as one of the armouries to prevent bacterial contamination. Verification of this hypothesis may lead to better understanding of means to prevent excessive bacterial contamination in WRF reactors; however, this is beyond the scope of the current study. Notably, due to the fragmentation of fungal pellets, possibly by bacterial protease (Leidig et al., 1999), the macroscopic morphology of the fungal biomass gradually changed from the pelleted to the dispersed form, and the enzymatic activity did not recover to the initial level within the remaining observation period.

In this study, the initial very high titre of enzymatic activity gradually decreased in the course of operation (Figure 1), especially after the disappearance of spongy pellets. In fact, by the end of the three month operation, the enzymatic activity in the reactor medium fell below the detection level (Figure 1). However, under the applied HRT (4.5 days), significant decolouration of the treated effluent continued. This observation is in line with available reports that while enzyme is indispensable, dye decolouration does not require very high enzymatic activity (Blanquez et al., 2008; Hai et al., 2012). However, it should be emphasized that maintenance of a stable expression of enzymatic activity, as opposed to a gradually diminishing activity, is required for a sustainable operation of bioreactors.

Faisal I. Hai, Kazuo Yamamoto, Fumiyuki Nakajima, Kensuke Fukushi, Long D. Nghiem, William E. Price, Bo Jin. Degradation 10 azo dye acid orange 7 in a membrane bioreactor by pellets and attached growth of Coriolus versicolour. Bioresource Technology, Volume 141, August 2013, Pages 29-34 


\subsection{Performance of the MBR with combined suspended and attached growth}

To date better efficiency and stability of attached growth biomass compared with the suspended pellets have been reported mainly in sterile batch tests (Rodriguez Couto, 2009) and in only a few studies concerning bioreactors operated under non-sterile conditions (Leidig et al., 1999; Nilsson et al., 2006; Tang et al., 2011). The aim of this part of the study was to verify our hypothesis that promotion of attached growth along with pellet formation would ensure greater resistance against bacterial inhibition of fungal enzymatic activity and decolouration performance than with growth only in pellet form.

\subsubsection{Sequencing batch mode}

In line with the initial expectations, in contrast to the growth solely in spherical pellets in the first reactor, both suspended (pellets) and attached growths (on the plastic tubes, the membrane module, and even onto the stirrer pedal) were observed in the stirred tank MBR. Furthermore, the size distribution of the suspended biomass was somewhat different in this reactor: in addition to a bit larger spherical pellets ( 0.7 as compared to $0.5 \mathrm{~cm})$, some larger, ellipsoidal clumps $(2 \times 3$ $\mathrm{cm}$ ) were observed floating in the reactor, possibly due to the different hydrodynamic conditions in the MBR in presence of additional components i.e., the membrane and the attached growth supports (tubes).

\section{Figure 2}

Similar to the previous trial, high enzymatic activity as well as excellent decolouration was observed right from the beginning (Figure 2). In addition, neither significant bacterial contamination nor reduction in decolouration performance was detected for 40 days of operation under non-sterile conditions in sequencing batch mode. This observation, when compared with the performance of the reactor with spongy pellets (Section 3.2), clearly shows better performance of the attached growth under non-sterile conditions. Our observation is in good agreement with a few other available studies who have reported better performance of immobilised fungus during non-sterile operation (Leidig et al., 1999; Nilsson et al., 2006; Tang et al., 2011). Interestingly, the spongy pellets were observed to gradually extend branches, bond Faisal I. Hai, Kazuo Yamamoto, Fumiyuki Nakajima, Kensuke Fukushi, Long D. Nghiem, William E. Price, Bo Jin. Degradation bf azo dye acid orange 7 in a membrane bioreactor by pellets and attached growth of Coriolus versicolour. Bioresource Technology, Volume 141, August 2013, Pages 29-34 
to each other and form networked masses. It is worth-noting here that poor long-term stability of aerobic granules is a widely-reported phenomenon (Liu \& Tay, 2004). However, unlike the case of pellet fragmentation due to bacterial contamination in the previous reactor (Section 3.2), the excellent enzymatic activity and decolouration performance continued despite the conversion of the pellets into larger irregular clumps. It is noteworthy that anoxic microniche in the internal layers of bacterial biofilm can significantly contribute to azo dye reduction (Venkata Mohan et al., 2012; Wang et al., 2012). It is possible that an anoxic microenvironment also prevails in the internal layers of the fungal attached growth. However, because WRF carry out dye degradation predominantly via oxidative enzymes under aerobic conditions, the contribution of anaerobic dye decoloration in this study is not expected to be significant. Further study in this line would be interesting; however, that is beyond the scope of this study.

\subsubsection{Continuous flow mode}

No significant change in enzymatic activity or decolouration performance was noticed following the change in operation to continuous flow mode at the same HRT. However, when the HRT was reduced to 1 day, the enzymatic activity in the reactor reduced significantly (Figure 3). Absence of any significant bacterial contamination at this point was confirmed by microscopic observation; therefore, the drop in enzymatic activity in the reactor could be attributed to significant direct loss of enzyme through the membrane under the shortened HRT. It is noteworthy that we have previously demonstrated the HRT-dependent impact of enzyme washout from WRF reactors, and have proposed effective measures (e.g., direct addition of activated carbon into MBR) to minimize enzyme washout (Hai et al., 2012; Hai et al., 2009). However, to allow study of the effect of morphology on dye degradation, such enzyme washout prevention approaches were not adopted here. Although reduced (due to washout of enzyme), the enzymatic activity in this reactor did not drop to undetectable level, possibly because the attached growth was intact and could continue to produce laccase. By contrast, in the reactor with only pellets (Section 3.2), the enzymatic activity gradually diminished due to fragmentation of pellets following bacterial contamination.

\section{Figure 3}

Faisal I. Hai, Kazuo Yamamoto, Fumiyuki Nakajima, Kensuke Fukushi, Long D. Nghiem, William E. Price, Bo Jin. Degradation $\mathbf{b F}$ azo dye acid orange 7 in a membrane bioreactor by pellets and attached growth of Coriolus versicolour. Bioresource Technology, Volume 141, August 2013, Pages 29-34 
To obtain additional evidence of the importance of the attached growth, the attached growth on the stirrer and the tubes was intentionally damaged by vigorously stirring the reactor. Following this, the enzymatic activity as well as decolouration performance gradually dropped. Simultaneous onset of severer bacterial contamination was also detected. This observation once again underscored the importance of agglomerated attached growth. A similar observation was made by Gao et al. (2009) who reported disappearance of peroxidases from the mixed liquor and a gradual reduction in dye decolouration efficiency along with the reduction of the coverage of fungal biofilm over the membrane surface.

It is noteworthy that membrane fouling can be different under suspended and attached growth conditions (Lee et al., 2001). However, a relatively low membrane flux was used in this study to avoid fouling. This allowed the focus of the study to remain on fungal dye removal performance (Section 2.3.2) and the transmembrane pressure remained stable throughout the observation period without any cleaning and irrespective of the suspended or attached growth conditions.

\subsection{TOC removal performance of the reactors}

TOC consumption in pure fungi culture proceeds much slower than that in a mixed culture of fungi and bacteria (Anastasi et al., 2012; Hai et al., 2009). Therefore, it is not surprising that TOC removal was rather low by the reactor with spherical pellets when fungal dominance was maintained (Figure 4). Only a moderate improvement in TOC removal performance (removal < $25 \%$ ) was observed even when bacterial contamination finally occurred in that reactor, indicating that the fungi still remained relatively dominant in that system. Better TOC removal, but still below $50 \%$, was achieved during the operation of the MBR (Section 3.3), particularly after intentional destruction of the attached growth, which triggered the onset of bacterial contamination.

In this study, colour removal was monitored by spectrophotometer and, additionally, the dye degradation was evidenced by HPLC-DAD analysis (Section 3.2 and Supplementary Data Figure 2). Because the dye contributed only $5 \%$ of the TOC of the wastewater, the extent of TOC consumption was not an indicator of dye degradation in this study. However, TOC removal is

Faisal I. Hai, Kazuo Yamamoto, Fumiyuki Nakajima, Kensuke Fukushi, Long D. Nghiem, William E. Price, Bo Jin. Degradation $b f$ azo dye acid orange 7 in a membrane bioreactor by pellets and attached growth of Coriolus versicolour. Bioresource Technology, Volume 141, August 2013, Pages 29-34 
important from the point of view of a complete treatment of the mixed wastewater because, from a textile mill, streams containing biodegradable organics in high concentration may originate in addition to the nutrient-deficient dye bath effluent that is relatively resistant to biodegradation (Hai et al., 2007).

\section{Figure 4}

Our results show that the maintenance of fungal dominance within the reactor allows stable enzymatic activity and excellent decolouration; however, this, on the other hand, hampers bacterial TOC removal. A subtle balance between fungal and bacterial dominance is necessary for simultaneous efficient decolouration and TOC removal. The contradictory requirements for decolouration and TOC removal indicate that it would be appropriate to use a two-step removal process - decolouration in a reactor with attached fungal growth followed by TOC removal completion in a reactor containing mixed microbial community. This approach is in line with a recent publication by Anastasi et al. (2012), who, however, did not focus on the aspect of WRF morphology. Following a systematic investigation, the current study puts forward a strategy for the first step (i.e. optimization of fungal decolouration process). Investigation on the combined process proposed above is currently underway and preliminary data show the technical feasibility of such an approach.

\section{Conclusions}

Spherical spongy pellets showed high enzymatic activity achieving efficient dye degradation initially. However, under extended non-sterile operation, irrecoverable bacterial damage of fungal pellets led to reduced enzymatic activity and decolouration. Simultaneous attached growth and pellet morphology provided greater resistance to bacterial inhibition of dye degradation. During continuous operation of an MBR containing attached growth and spherical pellets, although the level of enzymatic activity varied with the applied HRT (1-3 d), over 95\% decolouration could be achieved at an HRT of 1 day,. A bacterial post-treatment is suggested to improve the removal of TOC (mainly contributed by starch).

\section{Acknowledgement}

Faisal I. Hai, Kazuo Yamamoto, Fumiyuki Nakajima, Kensuke Fukushi, Long D. Nghiem, William E. Price, Bo Jin. Degradation $b f$ azo dye acid orange 7 in a membrane bioreactor by pellets and attached growth of Coriolus versicolour. Bioresource Technology, Volume 141, August 2013, Pages 29-34 
Dr. Faisal I. Hai acknowledges grants from the University Research Committee and the GeoQuEST Research Centre, respectively of University of Wollongong, Australia to carry out this work. A part of this study was carried out during Dr. Hai's tenure at The University of Tokyo, Japan with financial support from the Japan Society for Promotion of Science (JSPS).

Thanks are also due to Mitsubishi Rayon Co. Ltd., Japan for their supply of membrane modules.

\section{References}

[1] Anastasi, A., Spina, F., Romagnolo, A., Tigini, V., Prigione, V., Varese, G.C. 2012. Integrated fungal biomass and activated sludge treatment for textile wastewaters bioremediation. Bioresource Technology, 123, 106-111.

[2] Baldrian, P. 2004. Increase of laccase activity during interspecific interactions of white-rot fungi. FEMS Microbiology Ecology, 50, 245-253.

[3] Bermek, H., Guseren, I., Li, K., Jung, H., Tamerler, C. 2004. The effect of fungal morphology on ligninolytic enzyme production by a recently isolated wood-degrading fungus Trichophyton Rubrum LSK-27. World Journal of Microbiology and Biotechnology, 20, 345-349.

[4] Blanquez, P., Sarra , M., Vicent, T. 2008. Development of a continuous process to adapt the textile wastewater treatment by fungi to industrial conditions. Process Biochemistry, 43, 1-7.

[5] Borràs, E., Blánquez, P., Sarrà, M., Caminal, G., Vicent, T. 2008. Trametes versicolor pellets production: Low-cost medium and scale-up. Biochemical Engineering Journal, 42, 61-66.

[6] Erdal, S., Taskin, M. 2006. Uptake of textile dye Reactive Black-5 by Penicillium chrysogenum MT-6 isolated from cement-contaminated soil. African Journal of Microbiology Research 4, 618-625.

[7] Gao, D.W., Wen, X.H., Qian, Y. 2006. Decolorization of reactive brilliant red K-2BP by white rot fungus under sterile and non-sterile conditions. Journal of Environmental Science, 18, 428-432.

[8] Gao, S., Chen, C., Tao, F., Huang, M., Ma, L., Wang, Z., Wu, L. 2009. Variation of peroxidase isoenzyme and biofilm of Phanerochaete chrysosporium in continuous membrane bioreactor for Reactive Brilliant Red X3-B treatment. Journal of Environmental Sciences, 21, 940-947.

[9] Hai, F.I., Yamamoto, K., Fukushi, K. 2006. Development of a submerged membrane fungi reactor for textile wastewater treatment. Desalination, 192, 315-322.

[10] Hai, F.I., Yamamoto, K., Fukushi, K. 2007. Hybrid treatment systems for dye wastewater. Critical Reviews in Environmental Science and Technology, 37, 315-377.

[11] Hai, F.I., Yamamoto, K., Nakajima, F., Fukushi, K. 2012. Application of a GAC-coated hollow fiber module to couple enzymatic degradation of dye on membrane to whole cell biodegradation within a membrane bioreactor. Journal of Membrane Science, 389, 67-75.

[12] Hai, F.I., Yamamoto, K., Nakajima, F., Fukushi, K. 2009. Factors governing performance of continuous fungal reactor during non-sterile operation - The case of a membrane bioreactor treating textile wastewater. Chemosphere, 74, 810-817.

[13] Jimenez-Tobon, G.A., Penninckx, M.J., Lejeune, R. 1997. The relationship between pellet size and production of $\mathrm{Mn}$ (II) peroxidase by Phanerochaete chrysosporium in submerged culture. Enzyme and Microbial Technology, 21, 537-542.

[14] Lee, J., Ahn, W.-Y., Lee, C.-H. 2001. Comparison of the filtration characteristics between attached and suspended growth microorganisms in submerged membrane bioreactor. Water Research, $35,2435-2445$.

Faisal I. Hai, Kazuo Yamamoto, Fumiyuki Nakajima, Kensuke Fukushi, Long D. Nghiem, William E. Price, Bo Jin. Degradation bf azo dye acid orange 7 in a membrane bioreactor by pellets and attached growth of Coriolus versicolour. Bioresource Technology, Volume 141, August 2013, Pages 29-34 
[15] Leidig, E., Prusse, U., Vorlop, K.D., Winter, J. 1999. Biotransformation of poly R-478 by continuous cultures of PVAL- encapsulated Trametes versicolor under non-sterile conditions. Bioprocess Engineering, 21, 5-12.

[16] Libra, J.A., Borchert, M., Banit, S. 2003. Competition strategies for the decolorization of a textilereactive dye with the white-rot fungi Trametes versicolor under non-sterile conditions. Biotechnology and Bioengineering, 82, 736-744.

[17] Liu, Y., Tay, J.-H. 2004. State of the art of biogranulation technology for wastewater treatment. Biotechnology Advances, 22, 533-563.

[18] Nilsson, I., Moller, A., Mattiasson, B., Rubindamayugi, M.S.T., Welander, U. 2006. Decolorization of synthetic and real textile wastewater by the use of white-rot fungi. Enzyme and Microbial Technology, 38, 94-100.

[19] Papagianni, M. 2004. Fungal morphology and metabolite production in submerged mycelial processes. Biotechnology Advances, 22, 189-259.

[20] Rodriguez Couto, S. 2009. Dye removal by immobilised fungi. Biotechnology Advances, 27, 227-235.

[21] Tang, W., Jia, R., Zhang, D. 2011. Decolorization and degradation of synthetic dyes by Schizophyllum sp. F17 in a novel system. Desalination, 265, 22-27.

[22] Venkata Mohan, S., Suresh Babu, P., Naresh, K., Velvizhi, G., Madamwar, D. 2012. Acid azo dye remediation in anoxicâ€"aerobicâ€"anoxic microenvironment under periodic discontinuous batch operation: Bio-electro kinetics and microbial inventory. Bioresource Technology, 119, 362372.

[23] Wang, J., Liu, G.-F., Lu, H., Jin, R.-F., Zhou, J.-T., Lei, T.-M. 2012. Biodegradation of Acid Orange 7 and its auto-oxidative decolorization product in membrane-aerated biofilm reactor. International Biodeterioration \& Biodegradation, 67, 73-77.

[24] Wesenberg, D., Kyriakides, I., Agathos, S.N. 2003. White-rot fungi and their enzymes for the treatment of industrial dye effluents. Biotechnology Advances, 22, 161-187.

[25] Yang, S.F., Li, X.Y., Yu, H.Q. 2008. Formation and characterisation of fungal and bacterial granules under different feeding alkalinity and $\mathrm{pH}$ conditions. Process Biochemistry, 43, 8-14.

[26] Zhang, F.M., Knapp, J.S., Tapley, K.N. 1999. Development of bioreactor systems for decolorization of Orange II using white rot fungus. Enzyme and Microbial Technology, 24, 48-53.

[27] Zhou, C., Wen, X.H. 2009. Degradation of acid blue 45 in a white-rot fungi reactor operated under non-sterile conditions. Huanjing Kexue/Environmental Science, 30, 1797-1801.

[28] Žmak, P., Podgornik, A., Podgornik, H., Koloini, T. 2006. Impact of pellet size on growth and lignin peroxidase activity of Phanerochaete chrysosporium. World Journal of Microbiology and Biotechnology, 22, 1243-1249.

Faisal I. Hai, Kazuo Yamamoto, Fumiyuki Nakajima, Kensuke Fukushi, Long D. Nghiem, William E. Price, Bo Jin. Degradation 16 f azo dye acid orange 7 in a membrane bioreactor by pellets and attached growth of Coriolus versicolour. Bioresource Technology, Volume 141, August 2013, Pages 29-34 
Faisal I. Hai, Kazuo Yamamoto, Fumiyuki Nakajima, Kensuke Fukushi, Long D. Nghiem, William E. Price, Bo Jin. Degradation $\mathrm{b} 7$ azo dye acid orange 7 in a membrane bioreactor by pellets and attached growth of Coriolus versicolour. Bioresource Technology, Volume 141, August 2013, Pages 29-34 


\section{List of figures}

Figure 1 Colour removal and enzymatic activity profiles during non-sterile operation of the reactor initiated with spongy pellets. The reactor was operated at HRT ranging from 3 to $1 \mathrm{~d}$ for the first 26 days, and at an HRT of $4.5 \mathrm{~d}$ for the remaining period. Absorbance (colour) values of two times diluted samples have been plotted.

Figure 2 Colour removal and enzymatic activity profiles during non-sterile operation of the MBR in sequencing batch mode (HRT=3 to $4.5 \mathrm{~d}$ ). Absorbance (colour) values of two times diluted samples have been plotted.

Figure 3 Colour removal performance and enzymatic activity of the MBR initiated with spongy pellets and attached growth during non-sterile operation in continuous flow mode (HRT=4.5 to $1 \mathrm{~d}$ ). The same reactor was first operated in sequencing batch mode for $40 \mathrm{~d}$ (see Figure 2). Absorbance (colour) values of two times diluted samples have been plotted.

Figure 4 TOC removal during non-sterile operation of the reactor initiated with spongy pellets. The reactor was operated in sequencing batch mode at HRT ranging from 3 to $1 \mathrm{~d}$ for the first 26 days, and at an HRT of 4.5 days for the remaining period.

Faisal I. Hai, Kazuo Yamamoto, Fumiyuki Nakajima, Kensuke Fukushi, Long D. Nghiem, William E. Price, Bo Jin. Degradation 188 azo dye acid orange 7 in a membrane bioreactor by pellets and attached growth of Coriolus versicolour. Bioresource Technology, Volume 141, August 2013, Pages 29-34 


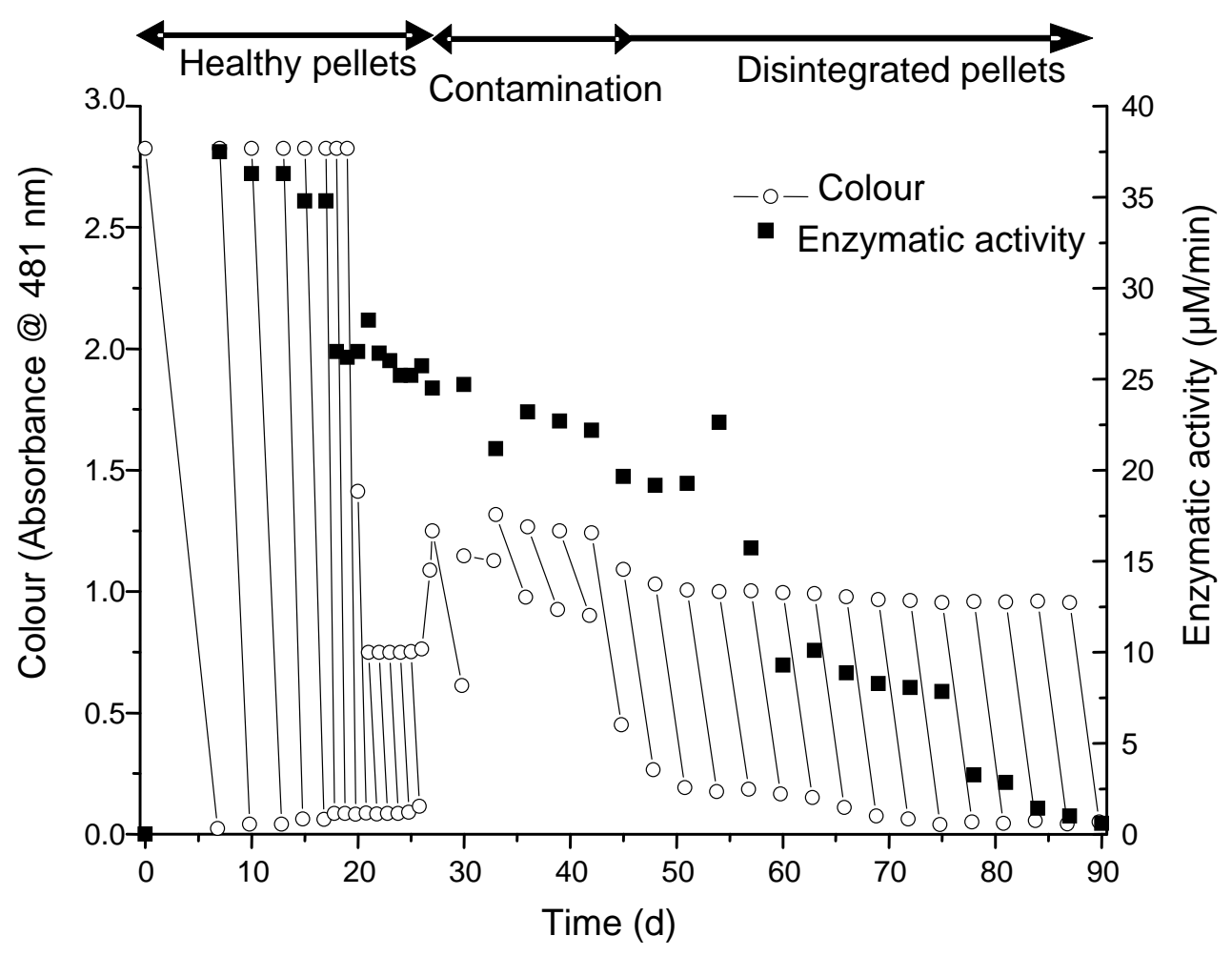

Figure 1

Faisal I. Hai, Kazuo Yamamoto, Fumiyuki Nakajima, Kensuke Fukushi, Long D. Nghiem, William E. Price, Bo Jin. Degradation $\mathbf{b f}$ azo dye acid orange 7 in a membrane bioreactor by pellets and attached growth of Coriolus versicolour. Bioresource Technology, Volume 141, August 2013, Pages 29-34 


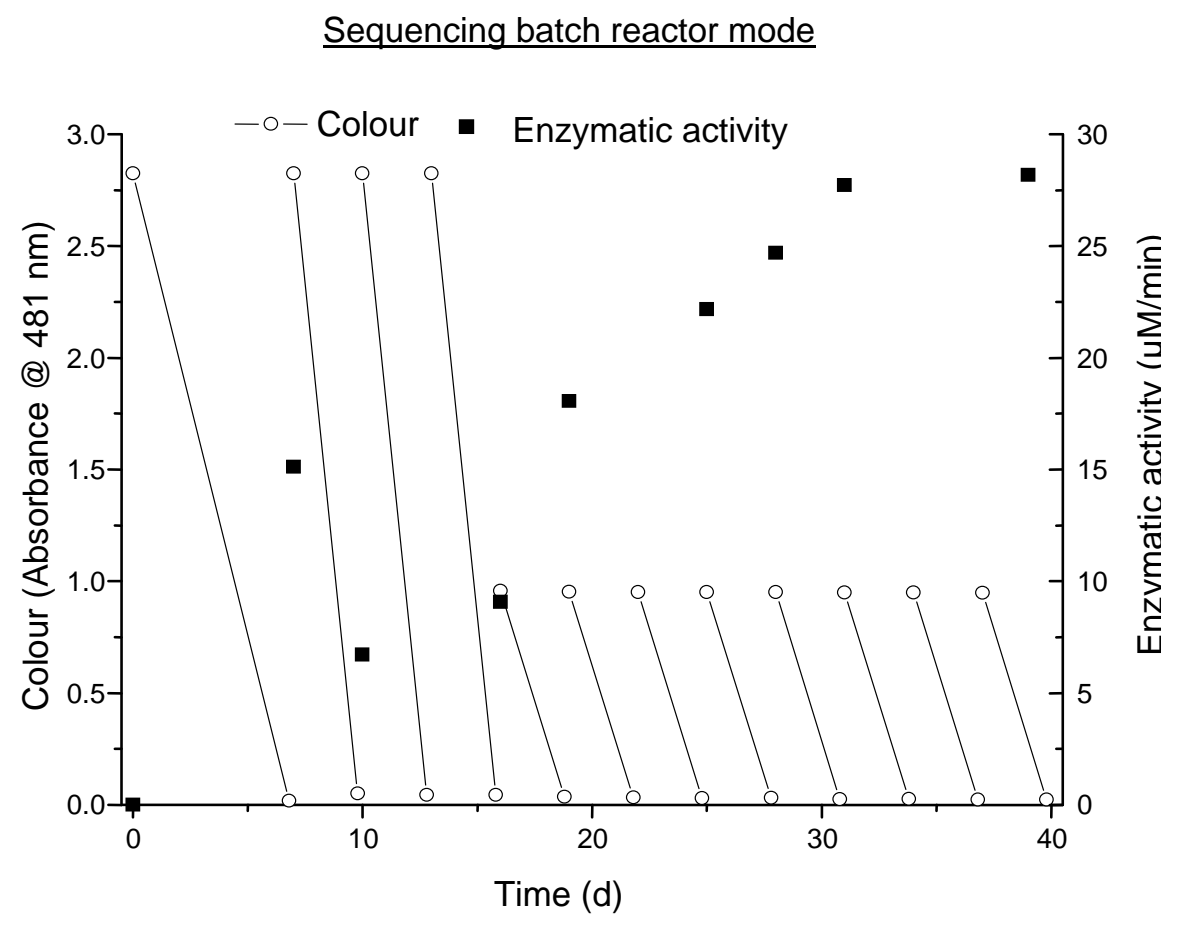

Figure 2

Faisal I. Hai, Kazuo Yamamoto, Fumiyuki Nakajima, Kensuke Fukushi, Long D. Nghiem, William E. Price, Bo Jin. Degradation 20 azo dye acid orange 7 in a membrane bioreactor by pellets and attached growth of Coriolus versicolour. Bioresource Technology, Volume 141, August 2013, Pages 29-34 


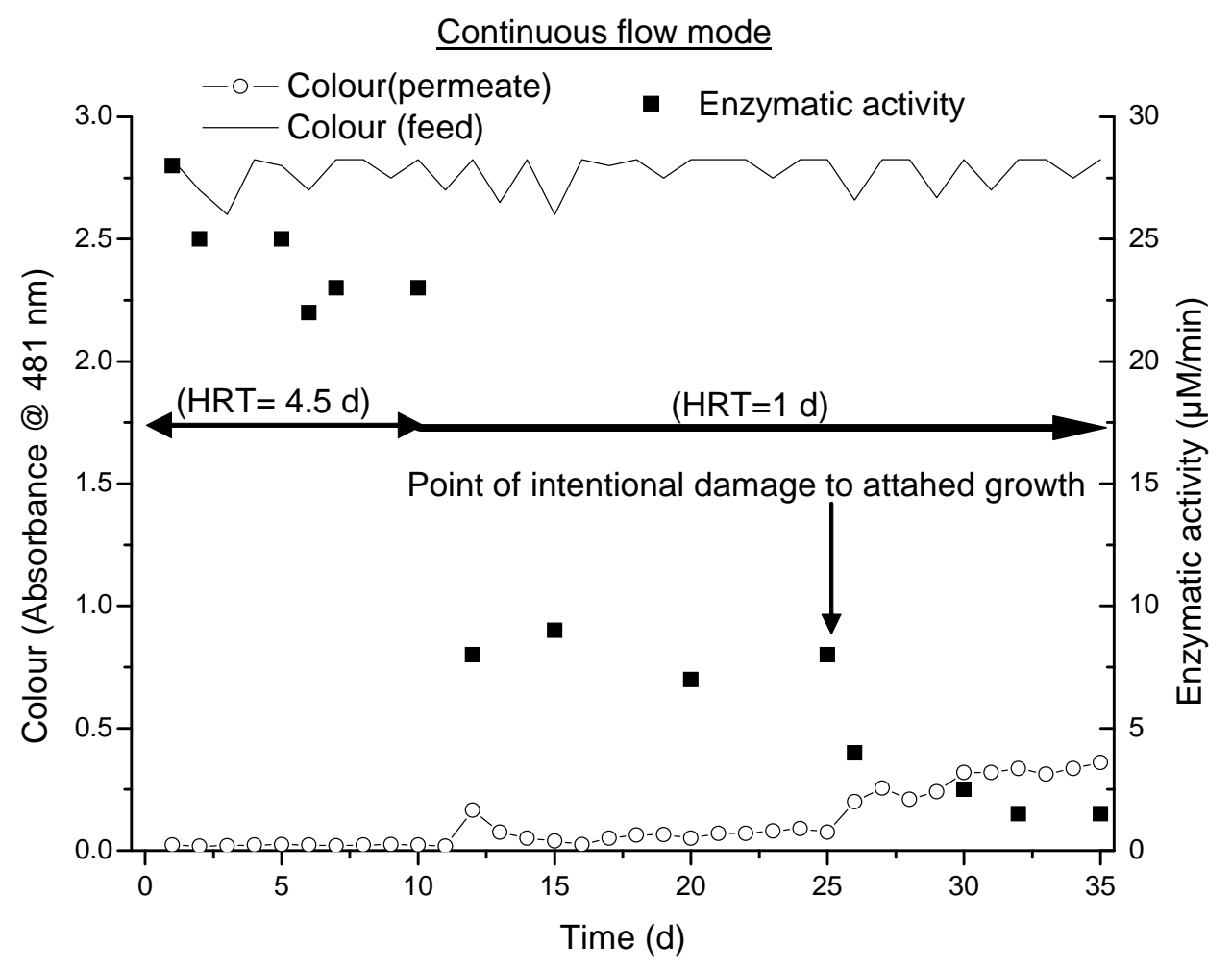

Figure 3

Faisal I. Hai, Kazuo Yamamoto, Fumiyuki Nakajima, Kensuke Fukushi, Long D. Nghiem, William E. Price, Bo Jin. Degradation zff azo dye acid orange 7 in a membrane bioreactor by pellets and attached growth of Coriolus versicolour. Bioresource Technology, Volume 141, August 2013, Pages 29-34 


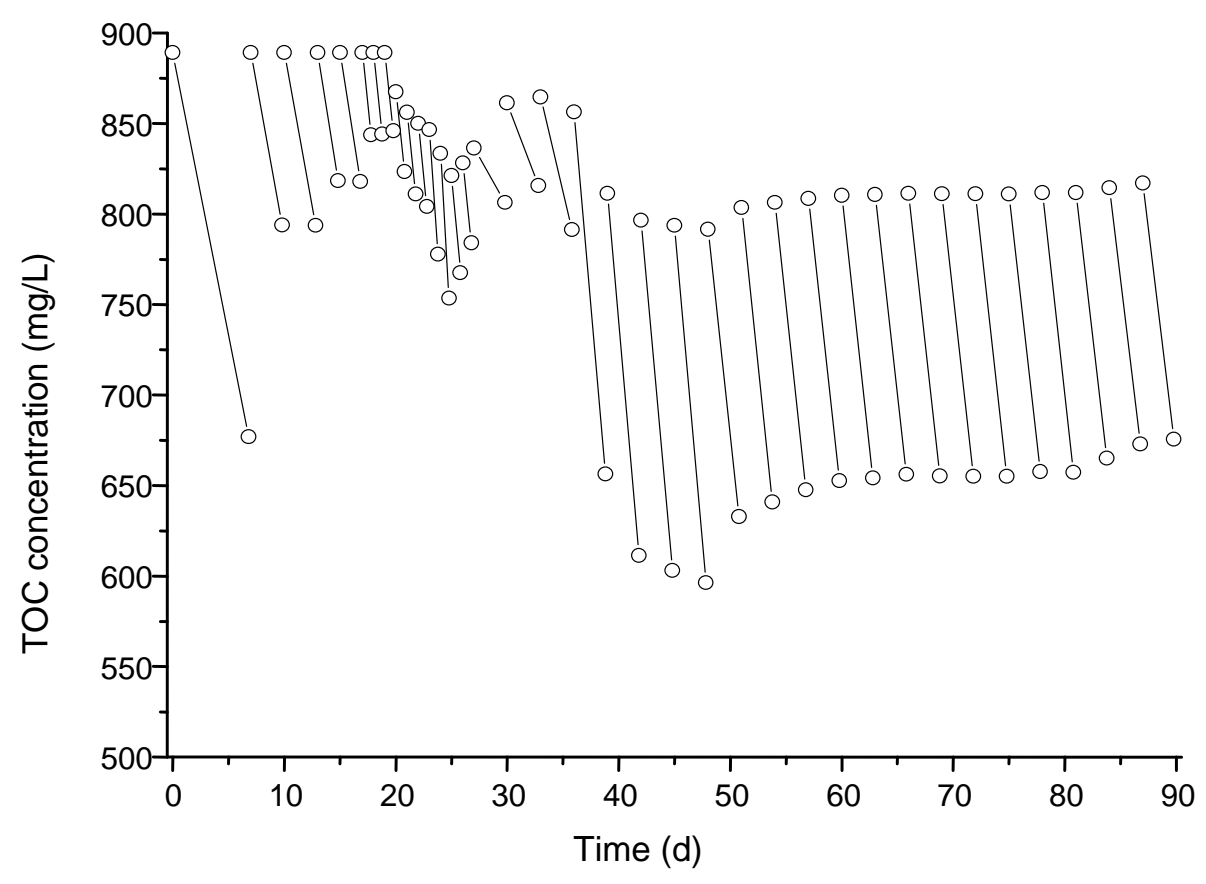

Figure 4

Faisal I. Hai, Kazuo Yamamoto, Fumiyuki Nakajima, Kensuke Fukushi, Long D. Nghiem, William E. Price, Bo Jin. Degradation $\mathbf{Z} \mathbb{F}$ azo dye acid orange 7 in a membrane bioreactor by pellets and attached growth of Coriolus versicolour. Bioresource Technology, Volume 141, August 2013, Pages 29-34 